\title{
Hyperbolic sets for twist maps
}

\author{
DANIEL L. GOROFF \\ Department of Mathematics, Harvard University, Cambridge, MA 02138, USA
}

(Received 9 September 1983 and revised 5 December 1984)

Abstract. An example is given of an area-preserving monotone twist map such that a uniformly hyperbolic structure exists on the closure of its Birkhoff maximizing orbits.

This note provides a rigorous example of an area preserving monotone twist map $f$ with the property that $\left.D f\right|_{\bar{B}}$ has a uniformly hyperbolic structure, where $\bar{B}$ denotes the closure of the Birkhoff maximizing orbits. As shown by Mather [8] and by Aubry, La Daeron, and André [3], the set $\bar{B}$ associated with $f$ contains invariant Cantor sets of all possible rotation numbers.

A result which would imply the hyperbolicity of these invariant Cantor sets was announced by Aubry in [1]. The heuristic justification given there is discussed further in [2]. Nevertheless, Katok raises the hyperbolicity question again in [4] and [5]. The construction below gives a rigorous answer based on an estimate first due to Aubry. Another proof that hyperbolic Cantor sets $\lambda$ can exist in $\bar{B}$ was obtained independently by Michel Herman.

Consider the 'standard' one parameter family of area preserving monotone twist maps of the cylinder $\mathbb{T}^{1} \times \mathbb{R}$. One lift to $\mathbb{R}^{2}$ of the map in this family corresponding to the parameter $k$ has the form

$$
f(x, y)=\left(x+y-\frac{k}{2 \pi} \sin 2 \pi x, y-\frac{k}{2 \pi} \sin 2 \pi x\right) .
$$

The function

$$
h\left(x, x^{\prime}\right)=-\frac{1}{2}\left(x-x^{\prime}\right)^{2}-\frac{k}{4 \pi^{2}} \cos 2 \pi x
$$

generates $f$ in the sense that $f(x, y)=\left(x^{\prime}, y^{\prime}\right)$ if and only if

$$
y=\frac{\partial h}{\partial x}\left(x, x^{\prime}\right) \text { and } y^{\prime}=-\frac{\partial h}{\partial x^{\prime}}\left(x, x^{\prime}\right) .
$$

Thus, given a sequence $\left\{x_{n}\right\}$, there exists a sequence $\left\{y_{n}\right\}$ such that $\left(x_{n}, y_{n}\right)=f^{n}\left(x_{0}, y_{0}\right)$ if and only if $\left\{x_{n}\right\}$ satisfies

$$
\frac{\partial h}{\partial x^{\prime}}\left(x_{n-1}, x_{n}\right)+\frac{\partial h}{\partial x}\left(x_{n}, x_{n+1}\right)=0
$$


in which case

$$
y_{n}=\frac{\partial h}{\partial x}\left(x_{n} x_{n+1}\right) \text {. }
$$

Following Birkhoff, this generating function for $f$ can be used to prove the existence of certain periodic orbits. Given integers $p$ and $q>0$, define the action $w: \mathbb{R}^{q} \rightarrow \mathbb{R}$ by

$$
w\left(x_{1}, \ldots, x_{q}\right)=\sum_{n=1}^{q} h\left(x_{n-1}, x_{n}\right)
$$

where $x_{0}+p=x_{q}$. It can be shown that the maximum of $w$ is achieved at a critical point, which therefore satisfies (1). If we associate second coordinates to this sequence using (2), the resulting union of $q$ points in $\mathbb{R}^{2}$ is called a Birkhoff maximizing orbit of type $(p, q)$ and its projection to $\nabla^{1} \times \mathbb{R}$ is a periodic trajectory. Let $B$ denote the union of all the orbits obtained in this way as $p$ and $q$ vary, and let $\bar{B}$ denote the closure of $B$ in $\mathbb{R}^{2}$.

For details concerning the construction and properties of $B$ and $\bar{B}$, see [3], [5], [6] and [8]. Although the last three references treat boundary preserving twist maps of an annulus, straightforward modifications allow the relevant results to apply to the standard family on the cylinder. See [7] in this regard.

LeMma (Aubry, [2]). Let $f$ be a standard map with parameter $k>2 \sqrt{1+\pi^{2}}$. If $\left(x_{0}, y_{0}\right)$ belongs to the set $B$ associated with $f$ then $-k \cos 2 \pi x_{0} \geq 2$.

Proof. Suppose $\left(x_{0}, y_{0}\right)$ belongs to a Birkhoff maximizing orbit of type $(p, q)$. By choosing an appropriate lift $f$, we can assume without loss of generality that $0<p / q \leq 1$. Let $\left(x_{n}, y_{n}\right)=f^{n}\left(x_{0}, y_{0}\right)$. Then $0<x_{n+1}-x_{n} \leq 1$ and, from (1), we have

$$
\left(x_{n+1}-x_{n}\right)-\left(x_{n}-x_{n-1}\right)+\frac{k}{2 \pi} \sin 2 \pi x_{n}=0 \text {. }
$$

This condition, which is independent of the lift chosen, implies $\left|\sin 2 \pi x_{n}\right| \leq 2 \pi / k$, and hence, for $k \geq 2 \pi$,

$$
\left|\cos 2 \pi x_{n}\right| \geq \sqrt{1-\frac{4 \pi^{2}}{k^{2}}} .
$$

Now from the definition of $B$ as sequences which maximize the function $w$, we also have

$$
\frac{\partial^{2} w}{\partial x_{n}^{2}}\left(x_{1}, \ldots, x_{q}\right)=-2+k \cos 2 \pi x_{n} \leq 0 .
$$

For $k>2 \sqrt{1+\pi^{2}},(3)$ and (4) together ensure that

$$
-k \cos 2 \pi x_{n} \geq k \sqrt{1-\frac{4 \pi^{2}}{k^{2}}}>2,
$$

which completes the proof.

THEOREM. Let $f$ be a standard map with parameter $k>2 \sqrt{1+\pi^{2}}$. Then the set $\bar{B}$ associated with $f$ has a uniform hyperbolic structure. 
Proof. Using the characterization of hyperbolicity given by Newhouse and Palis [10], it suffices to find a cone $C$ in $\mathbb{R}^{2}$ and a positive integer $m$ such that for each $(x, y)$ in $\bar{B}$, the derivative of $f$ at $(x, y), D_{(x, y)} f$, maps $C$ into itself and such that $D_{(x, y)} f^{m}$ expands $C$ and $D_{(x, y)} f^{-m}$ expands $\mathbb{R}^{2} \backslash C$ (see also [9]).

From the lemma, we have that for $(x, y)$ in $\bar{B}$,

$$
D_{(x, y)} f=\left[\begin{array}{cc}
1-k \cos 2 \pi x & 1 \\
-k \cos 2 \pi x & 1
\end{array}\right]=\left[\begin{array}{cc}
1+\varepsilon_{0} & 1 \\
\varepsilon_{0} & 1
\end{array}\right]
$$

for some $\varepsilon>2$. Let $C=\left\{\left(v_{1}, v_{2}\right) \in \mathbb{R}^{2} \mid v_{1} v_{2} \geq 0\right\}$. Then $D_{(x, y)} f$ not only maps each $v$ in $C$ back into $C$, but also expands its Euclidean norm in the sense that

$$
\left\|D_{(x, y)} f[v]\right\| \geq \sqrt{2}\|v\|
$$

for $v$ in $C$. Similarly, we find

$$
D_{(x, y)} f=\left[\begin{array}{cc}
1 & -1 \\
-\varepsilon & 1+\varepsilon
\end{array}\right],
$$

for some $\varepsilon>2$, and so

$$
\left\|D_{(x, y)} f[v]\right\| \geq \sqrt{5}\|v\|,
$$

whenever $v$ belongs to $\mathbb{R}^{2} \backslash C$. This completes the proof.

The author thanks John Mather and Michel Herman for their helpful comments, and gratefully acknowledges the support of the Institut des Hautes Etudes Scientifiques during the 1982-1983 academic year.

\section{REFERENCES}

[1] G. André \& S. Aubry. Annals of the Israel Physical Society 3 (1979), 133-164.

[2] S. Aubry. The twist Map, the extended Frenkel-Kontorova model and the Devil's staircase. Physica D. 7 (1983), 240-258.

[3] S. Aubry, P. Y. Le Daeron \& G. André, Classical ground-states of a one-dimensional model for incommensurate structures. Submitted for publication to Communications in Mathematical Physics.

[4] A. Katok. Problem list of special session on differential geometry and ergodic theory in Amherst. Preprint, 1981.

[5] A. Katok. Some remarks on Birkhoff and Mather twist map theorems. Ergod. Th. \& Dynam. Syst. 2 (1982), 185-194.

[6] J. N. Mather. Existence of quasi-periodic orbits for twist homeomorphisms of the annulus. Topology 21 (1982), 457-467.

[7] J. N. Mather. Non-existence of invariant circles. Ergod. Th. \& Dynam. Sys. To appear.

[8] J. N. Mather. A criterion for the non-existence of invariant circles. Preprint, 1982.

[9] S. E. Newhouse. Lectures on Dynamical Systems. In J. Guckenheimer, J. Moser \& S. E. Newhouse. Dynamical Systems: C.I.M.E. Lectures. Birkhauser, 1980, pp. 1-114.

[10] S. E. Newhouse \& J. Palis. Bifurcations of Morse-Smale dynamical systems. In Dynamical Systems (M. Peixoto, ed.). Academic Press, 1973, pp. 303-366. 